\title{
Téoros
}

Revue de recherche en tourisme

\section{La géographie du tourisme gourmand}

\section{Gérard Beaudet}

Volume 25, numéro 1, printemps 2006

Entre la culture du goût et le goût de la culture

URI : https://id.erudit.org/iderudit/1071027ar

DOI : https://doi.org/10.7202/1071027ar

Aller au sommaire du numéro

Éditeur(s)

Université du Québec à Montréal

ISSN

0712-8657 (imprimé)

1923-2705 (numérique)

Découvrir la revue

Citer cet article

Beaudet, G. (2006). La géographie du tourisme gourmand. Téoros, 25(1), 10-14. https://doi.org/10.7202/1071027ar d'utilisation que vous pouvez consulter en ligne.

https://apropos.erudit.org/fr/usagers/politique-dutilisation/ 


\section{La géographie du tourisme gourmand}

\section{Gérard Beaudet}

Mais déjà, à Rouaine, au Relais des Bécassiers, on vous sert un gratin de navets d'un autre siècle, aussi éloigné du premier McDo, que Saint-Armand peut l'être du Kosovo ou de Darhmensala.

Pierre Foglia

De nos jours, les valorisations touristiques, à l'instar des valorisations patrimoniales et paysagères, se déclinent au pluriel. Le tourisme est désormais urbain, rural, agricole, maritime, littoral, industriel, religieux, sacré, sexuel, patrimonial, de santé, de montagne, de grande nature ou d'aventure, quand il n'est pas écotourisme, cyclotourisme, agrotourisme.

Même si certaines formes de tourisme innovent quant à leur objet, leur espace d'inscription ou l'expérience proposée, bon nombre de ces émergences tiennent davantage d'initiatives qui mettent l'accent sur l'une ou l'autre des dimensions usuelles de cette activité. C'est le cas du tourisme gourmand. La découverte des traditions culinaires des contrées visitées, ainsi que les plaisirs gastronomiques proposés par les établissements hôteliers et les restaurants des capitales, des métropoles et des autres villes, ont en effet toujours fait partie de l'expérience touristique.

Ce qui, en la circonstance, distingue le tourisme gourmand des formes usuelles de découvertes culinaires paraît donc tenir plus de la place qu'occupe cette activité à l'intérieur d'une expérience touristique spécifique que d'une transformation de la nature intrinsèque de cette dernière. Ce report d'accent s'inscrit incidemment dans le sillage d'une autonomisation progressive de la

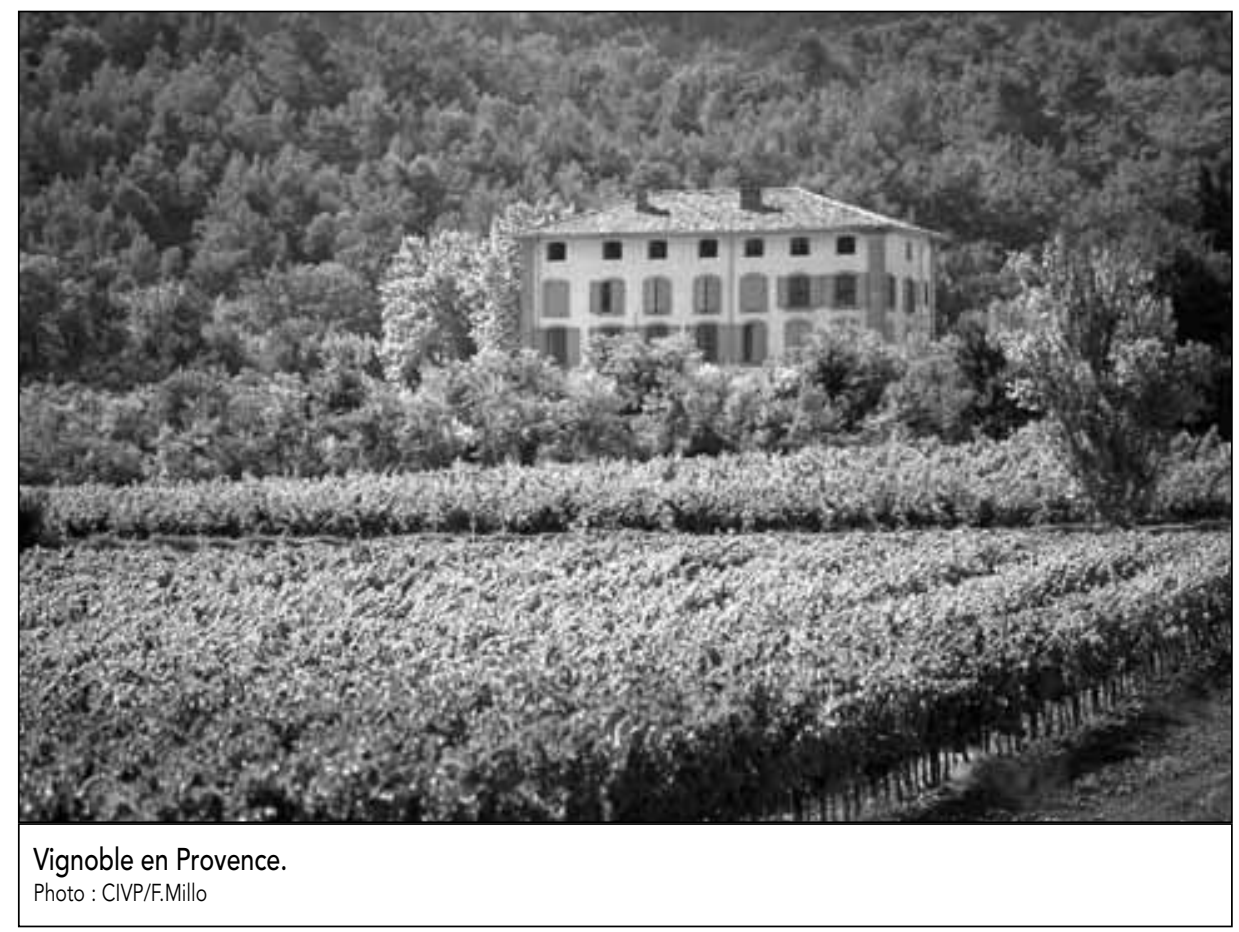

composante alimentaire du voyage et du séjour, ce dont rend notamment compte la transformation des guides touristiques. Si, à l'origine, ceux-ci conféraient aux renseignements concernant la restauration un caractère d'abord utilitaire, un peu comme le feront les guides qui proposeront aux pionniers du tourisme automobile une liste de garages, les pratiques évolueront rapidement dans le sens d'une forme de certification qualitative. C'est ainsi que le guide Michelin fournira, dès le milieu des années 1920, des indications concernant la qualité de la table proposée par certains établissements. La critique gastronomique, dont la paternité est attribuée à Alexandre Balthazar Grimod de la Reynière (1758-1838), ci-devant auteur de l'Almanach des gourmands paru une première fois en 1803, se lançait dès lors à l'assaut de l'espace géographique en alliant expérience gastronomique et découverte touristique.
La multiplication, au cours du dernier demisiècle, de guides comportant des renseignements de nature agroalimentaire et d'autres qui seront entièrement consacrés à ce sujet - dont le GaultMillau hôtels et restaurants, publié à compter de 1972, le Bottin gourmand, édité depuis 1982, et le Guide des gourmands, disponible depuis 1988 anticipera, puis accompagnera de manières diverses, l'émergence du tourisme gourmand. En France, l'offre de passeports gourmands poursuit sur cette lancée. L'utilisation du terme passeport suggère même l'association formelle d'un territoire à une activité gastronomique. À y regarder de plus près, on se rend toutefois compte qu'il s'agit essentiellement d'une régionalisation qui tient moins à une géographie donnée des traditions alimentaires qu'à une commodité de regroupement des établissements proposés. 
Le report de l'accent sur une dimension spécifique de l'expérience touristique n'est malgré tout pas banal, dans la mesure où il participe d'une spécialisation et d'une reconfiguration généralisée de l'offre touristique que caractérisent le façonnement de produits d'appel de niches, la multiplication des «labellisations » et la mise en œuvre de stratégies de marketing territorial de plus en plus étroitement ciblées. C'est d'ailleurs ce dernier aspect de la mise en tourisme actuelle que nous aborderons dans le présent article. En d'autres termes, nous tenterons de répondre à la question: le tourisme gourmand se définit-il par un espace géographique qui lui est propre?

\section{La dimension spatiale du tourisme gourmand}

Le tourisme gourmand est avant tout une expérience culinaire. Rien, dans cette désignation, ne suggère une quelconque affiliation géographique, contrairement en cela à d'autres produits, par exemple le tourisme littoral ou de montagne, ou encore l'agrotourisme. Le tourisme gourmand semble d'ailleurs se pratiquer aussi bien dans un grand restaurant d'une capitale qu'à la ferme, dans une auberge de village que dans un vignoble, voire dans un abri sommaire au sommet d'une montagne ou sur une péniche.

II n'en reste pas moins que cette diversité des cadres géographiques d'inscription du tourisme gourmand occulte en partie une connotation spatiale de plus en plus répandue qui fait du terroir, sinon le lieu de pratique privilégié du tourisme gourmand, à tout le moins l'aire de provenance des denrées alimentaires qu'il met en œuvre. En d'autres termes, si le tourisme gourmand ne se pratique pas qu'à la campagne, celle-ci en constituerait le terreau privilégié, tout en fournissant aux chantres urbains de cette forme de tourisme une caution vertueuse :

S'il y a une chose qui distingue Québec de Montréal ou de toute autre grande ville canadienne, c'est bien le lien intime qui unit chefs de cuisine et producteurs artisans. Cette union leur permet de développer ensemble le fameux concept de la terre à la table, qui est à la base même du label de terroir et qui garantit en partie son authenticité. (Desjardins, 2005)
Mais, on le verra, le terroir dont il est question ici tient souvent moins d'une conception rigoureuse des modalités d'occupation et d'aménagement de l'espace géographique que d'une certaine idéalisation de la campagne, quand ce n'est pas d'une récupération à des fins de positionnement et de promotion économiques.

\section{Goûter le pays}

II existe plusieurs définitions du terroir. Tantôt associé à une unité physique de taille variable dotée d'un certain potentiel agricole (terroir de fond de vallée, de versant de montagne, de plaine, de butte, etc.), le terme renvoie en d'autres circonstances à un espace aménagé dont les caractères résultent de l'effet combiné du potentiel des sols et des pratiques culturales adoptées par les populations locales (terroir irrigué, en terrasse, viticole, etc.). Si le terme a surtout été utilisé par les géographes pour désigner une étendue de pays qui présente une certaine homogénéité biogéographique et agronomique, il a aussi été retenu pour rendre compte par extension de particularités culturelles locales, notamment langagières, religieuses, architecturales. Enfin, le terme peut signaler une filiation avec un habitat et un environnement socioculturel donnés ; c'est ainsi qu'on parlera de littérature du terroir.

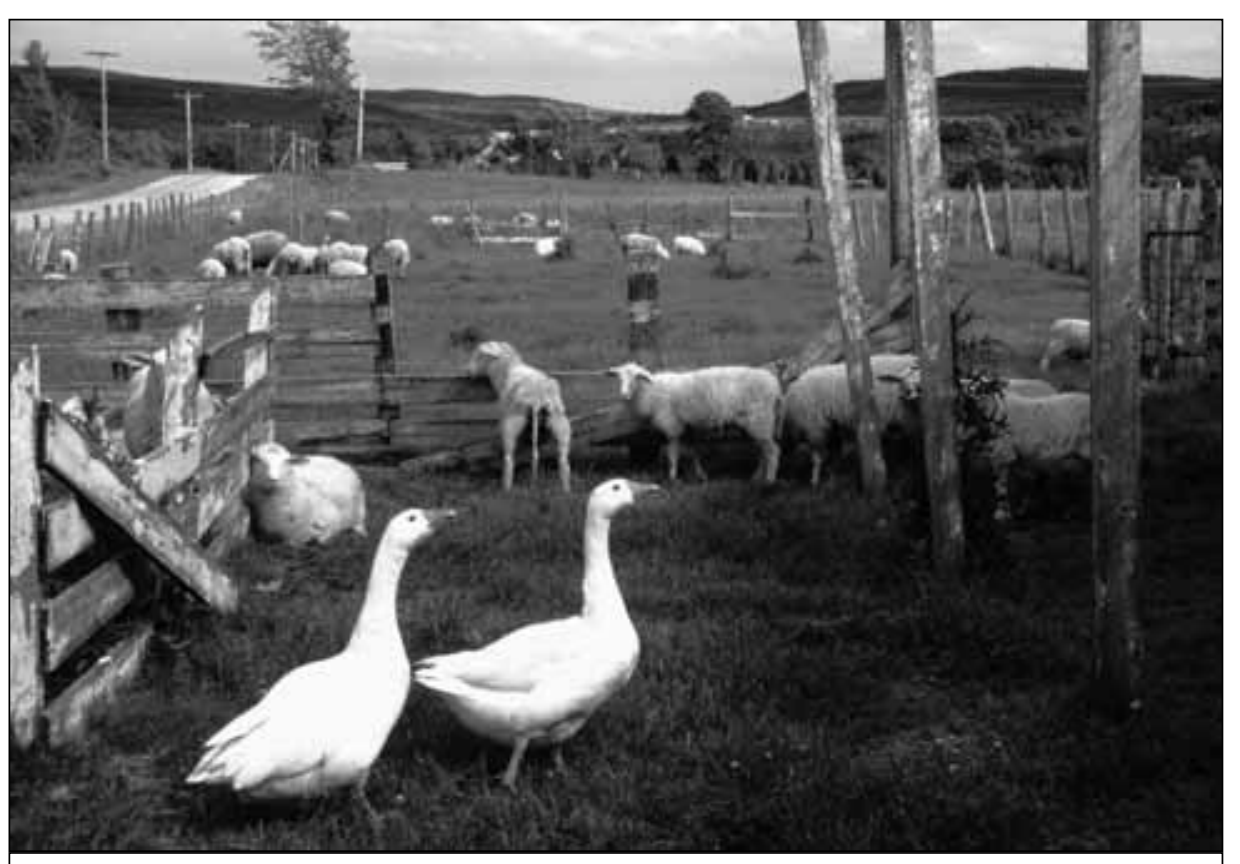

Oies et moutons de la Bergerie La Moutonnière, Sainte-Hélène de Chester (Québec).

Photo : @ Tourisme Québec

Le terme a par ailleurs connu des fortunes diverses. II a notamment connoté en certaines circonstance, particulièrement au milieu du siècle dernier, au moment où nos sociétés amorçaient une nouvelle phase de modernisation, ce qui était assimilé à des défauts partagés par les habitants d'un lieu, par exemple un fort accent ou certains archaïsmes culturels. De nos jours, le regain d'intérêt pour le terroir apparait étroitement lié à la mondialisation. II révélerait autant une saine résistance à l'érosion des cultures et des économies locales qu'une affirmation d'appartenances et d'ancrages identitaires porteurs d'avenir. Ce faisant, il a acquis une acceptation presque morale.

La popularité croissante du terme s'accompagne toutefois d'une certaine dilution de sa signification. Originellement, le terroir désignait une entité géographique émergente, fondée sur la convergence, dans la longue durée, de potentialités biogéographiques et de pratiques culturales. En Europe, les terroirs ont ainsi été le résultat de plusieurs siècles d'occupation du territoire rural, leur construction étant marquée par les variations climatiques, l'évolution des techniques agraires, des temps longs de stabilité, des moments de bouleversements parfois radicaux (les guerres, les épisodes de la peste noire), l'influence de certains acteurs (les communautés monastiques), l'attractivité des marchés urbains, la transformation des 
pratiques alimentaires (la démocratisation de la viande) et les influences extérieures.

En revanche, de nos jours, le terroir est souvent synonyme de campagne, de territoire des traditions, voire d'aire de production artisanale, sans nécessairement que ce caractère en apparence distinctif s'inscrive dans la longue durée ou qu'il corresponde à un espace géographique bien déterminé. Cela est particulièrement vrai au Québec, où le concept n'a jamais connu une diffusion étendue et où les élevages aussi bien que les pratiques culturales dont il est question peuvent être relativement récentes. Le terroir connote en la circonstance moins une réelle émergence conditionnée par le milieu qu'une adaptation d'emprunts à un espace géographique donné, dont l'influence peut, au demeurant, être passablement négligeable.

Certes, l'importation de produits alimentaires, de cultures ou d'élevages n'est pas l'apanage de nos sociétés actuelles. La route des épices aura constitué, voilà quelque 3500 ans, une des plus durables manifestations de la mondialisation des échanges entre les groupes humains (Caldicott, 2001). Et on ne compte plus les espèces végétales et animales qui ont été introduites dans des milieux dont elles avaient été totalement absentes, notamment à la suite des grandes découvertes de la Renaissance, et qui ont subséquemment été absorbées dans une véritable culture du terroir. II n'en reste pas moins que les brassages de populations et la réduction des contraintes traditionnellement associées aux distances et aux obstacles géographiques ont, plus que jamais auparavant, favorisé les métissages agroalimentaires, qui ont peu à voir avec une véritable dynamique de terroirs. Mentionnons, pour illustrer le propos, la région de Montréal, où plusieurs agriculteurs et éleveurs ont misé, depuis quelques années, sur des productions qui visaient à répondre à une demande en provenance de restaurants ethniques ou des immigrants qui fréquentent les marchés publics et les épiceries spécialisées. Parler en la circonstance de productions du terroir équivaudrait à conférer à celles-ci des attributs qu'elles n'ont pas, du moins pas encore.

L'ouvrage de Jacques Dorion (2003) sur les terroirs du Québec illustre bien cette tendance. Bien que l'auteur souligne avec à-propos que le terroir québécois a une longue histoire, il n'en reste pas moins que ce dont il est question dans cet ouvrage tient souvent plus de productions géographiquement localisées que de véritables terroirs. C'est ce dont témoignent certains passages de l'introduction où il est notamment question de paysages gourmands du Québec, qui «sont ceux de quatorze régions agrotouristiques et de leur terroir ", l'emploi du singulier suggérant une adéquation entre les premières (un découpage essentiellement administratif) et les seconds. Le circuit des paysans développé dans le sud-ouest de la Montérégie, de même que la route gourmande des fromages fins du Québec, qui regroupe plus de 60 producteurs répartis dans toutes les régions du Québec, évoquent une telle acceptation du concept de terroir.

Émergence culturelle dans un cadre géographique particulier ou plus prosaïquement référence spatiale utilitaire, le terroir apparaît donc être en mesure de cautionner avec une efficacité certaine la qualité des productions dont on cherche à faire la promotion. Mais il est de plus en plus évident que les possibilités de faire goûter le pays en font saliver plus d'un. Comment s'étonner dès lors que les demandes d'adoption de règles claires pour assigner les appellations réservées, destinées à garantir la qualité et l'authenticité d'une production, se fassent de plus en plus pressantes?

Les initiatives de valorisation ne se situent toutefois pas sur le seul terrain des appellations réservées. La dimension paysagère de la caution terroir semble en effet occuper une place de premier choix dans les stratégies de nombreux promoteurs du tourisme gourmand.

\section{Regardez comme c'est bon !}

Même s'il mobilise d'emblée le goût, il est indéniable que le tourisme gourmand propose, à l'instar des formes antérieures de la restauration, une expérience plurisensorielle. Odorat, vue, ouie et toucher sont en effet directement et diversement sollicités. L'espace de cette sollicitation est évidemment celui de la table et de son environnement immédiat. II n'y est toutefois pas nécessairement confiné. Plusieurs établissements offrent à leur clientèle des ambiances et des points de vue plus ou moins étendus. Tantôt à caractère intimiste (la cour intérieure d'un bâtiment urbain, d'un ensemble agricole, d'un monastère ou d'un château, la place urbaine ou de village), cette vue et cette ambiance peuvent en d'autres circonstances embrasser et s'inscrire dans de vastes panoramas, que ce soit à la montagne, sur le littoral ou à la campagne. Cela n'est évidemment pas inédit.
Ce qui l'est, en revanche, réside pour une bonne part dans les nouvelles avenues qu'emprunte la sollicitation des sens, en particulier la vue. Les productions du terroir et les traditions culinaires sont en effet de plus en plus fréquemment et explicitement évoquées par des paysages. On ne compte d'ailleurs plus les ouvrages grand public qui proposent tantôt des productions agricoles, viticoles ou animalières, tantôt des recettes du terroir, du pays ou régionales, en les associant à des paysages. Le beau livre de H. Walden (1993) Régions gourmandes: les pays de la Loire, paru originellement en Grande-Bretagne en 1992, en constitue un excellent exemple. On y trouve en effet autant des descriptions biogéographiques que des notes historiques, des références sur les traditions culturales que des recettes, le tout agrémenté de nombreuses photographies qui évoquent indistinctement les différents sujets abordés.

Certes, la géographie a depuis longtemps établi des liens entre terroir et paysage. On ne s'en étonnera guère quant on sait que le paysage du géographe est tout bonnement une étendue variable de pays qui s'offre à la vue. Le paysage dont il est question ici ne doit toutefois pas être confondu avec celui des géographes. II s'agit plutôt d'une invention (Cauquelin, 1989 ; Roger, 1997) d'une construction sociale sans cesse renouvelée et alimentée par les sensibilités artistiques et littéraires.

La mise en paysage du pays passerait donc par la construction, à un moment donné de l'histoire d'un groupe social, d'une métaphore paysagère, par une élite littéraire, un peintre, ou tout autre artiste susceptible, grâce à son rayonnement, de permettre la diffusion de cette image dans l'ensemble de la société - locale puis globale -, c'est-àdire de la socialisation d'un paysage. (Luginbülh, 1995 : 325)

L'exposition Sous le soleil, exactement: Le paysage en Provence, du classicisme à la modernité (1750-1920), tenue au musée des Beaux-Arts de Montréal en 2005, le film Sous le ciel de Toscane de la réalisatrice Audrey Wells (2003), le best-seller de Peter Mayle (1991) A Year in Provence, ainsi que le beau livre de Jacques Dorion (2003) Un dimanche à la campagne, constituent autant d'exemples de cette construction. 
Le paysage est donc le produit d'un regard par définition sélectif. Si, comme le soutient Pierre Donadieu, (1999: 85) « la campagne est un paysage avant d'être un lieu de production ", ce ne sont pas toutes les campagnes qui peuvent aspirer à un tel statut. Les campagnes assujetties à des pratiques culturales industrialisées ou à des élevages intensifs qui comportent leur lot de nuisances environnementales échappent en effet à cette catégorisation.

En fait, [les] nouveaux résidents [...] sont en train de parfaire l'œuvre commencée il y a quelques siècles: s'emparer d'un paysage qui a été produit pour l'essentiel par la société paysanne, qui en conserve les teintes, mais recomposée dans une représentation écartant la réalité moderne de l'agriculture, refusant le remembrement et les bâtiments d'élevage, maintenant des formes dépassées par les techniques actuelles ou rayées de l'espace par les tendances de l'économie ou les règlements des politiques agricoles nationales ou communautaires. (Luginbülh, 1995 : 320)

En conséquence, pour qu'une campagne devienne paysage, il lui faut être pittoresque. II lui faut également être porteuse de mémoire et être évocatrice de traditions, y compris, de plus en plus, de traditions culinaires. Le développement de l'agrotourisme, des tables champêtres et des routes à thème centrées sur les productions du terroir ont contribué, au cours des dernières années, à associer étroitement paysage et plaisirs gourmands. Tout se passe comme si la qualité du paysage donnait à voir la qualité et la renommée du produit ou du repas. C'est notamment ce que révèlent les ouvrages de deux auteurs chez qui la passion pour le vin se double d'une sensibilité certaine pour les paysages des vignobles.

Lorsqu'il s'agit de vin, l'influence du terroir s'impose avec davantage d'évidence encore. Le goût du vin peut être "sublimé " par les paysages de son terroir que nos souvenirs font resurgir dès la première gorgée. (Pigeat, 2000: 16)

Des douces collines de la Côte d'Or aux spectaculaires terrasses du Douro en passant par les incomparables châteaux de Bordeaux et les cornes d'abondance que sont devenus les vignobles du Nouveau Monde, c'est tout un formidable jardin conquis grâce à la ténacité et à la soif des hommes. Autant le vin nous comble par son goût raffiné, autant il impressionne le voyageur par la beauté de son univers. (Phaneuf, 2005: 7)

La relation entre paysage et production alimentaire n'est cependant pas univoque. L'introduction de nouvelles pratiques culturales peut également alimenter la construction paysagère, comme dans la région de Brome-Missisquoi, au sud de Montréal, où les vignobles se sont multipliés depuis une vingtaine d'années. Non seulement la mise en terre de vastes plages de vignes transforme-t-elle le substrat biogéographique à partir duquel se construit le paysage, mais elle favorise la construction paysagère en donnant en quelque sorte ses lettres de noblesse à un terroir émergeant et doté, au demeurant, d'une valeur patrimoniale manifeste. L'invitation à parcourir la route des vins est ici incitation au plaisir des sens.

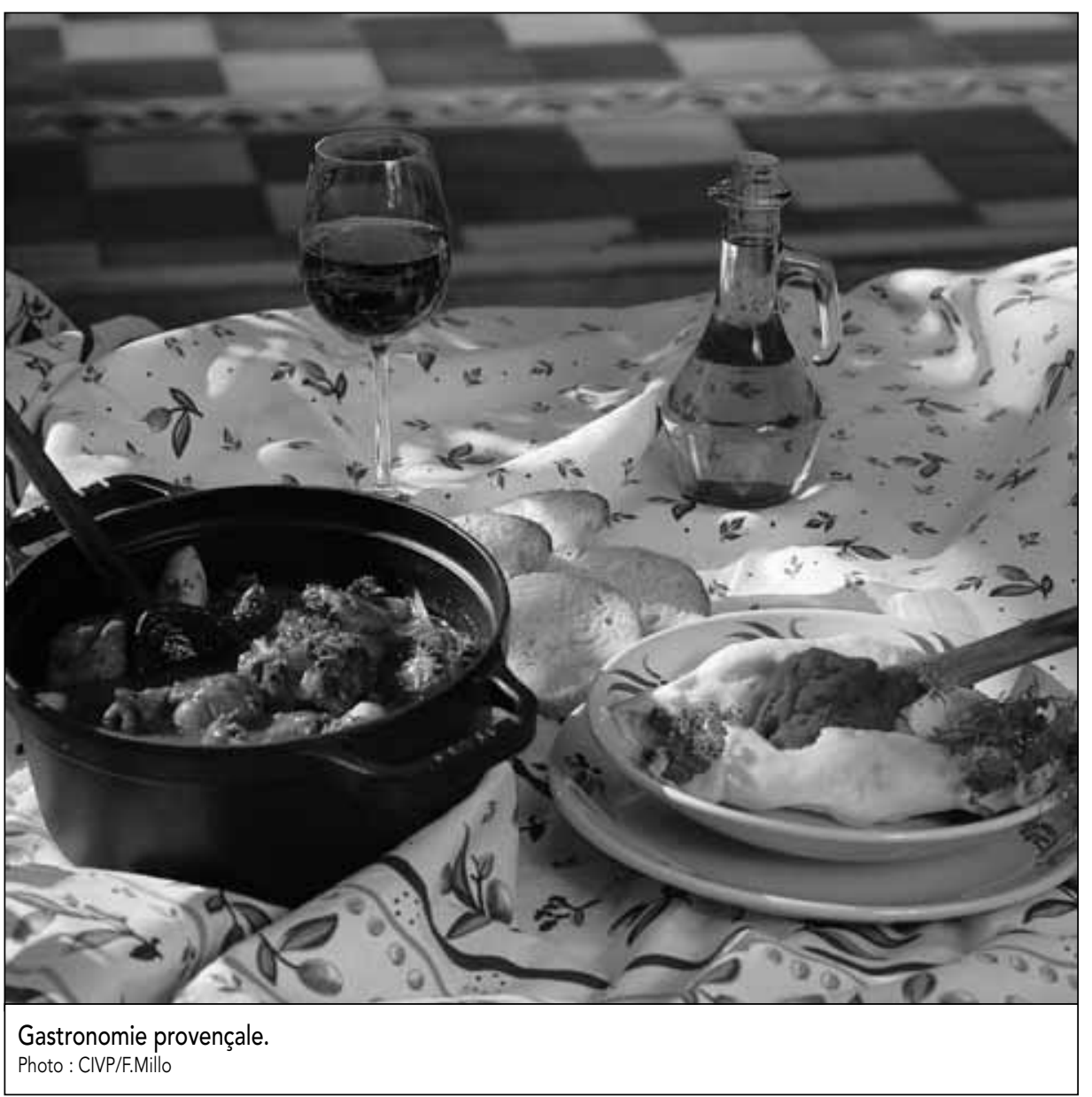

\section{Terroir, positionnement économique et marketing territorial}

Cette symbiose croissante participe du processus de construction des nouvelles campagnes urbaines dont Pierre Donadieu (1998) a révélé les fondements. En d'autres termes, elle constitue un des piliers de la différenciation qualitative des campagnes par la construction paysagère. II y aurait les campagnes urbaines, qui méritent l'appellation en apparence contradictoire de terroir, et il y aurait les autres, industrialisées, qui seraient abandonnées à un productivisme désincarné.

Les enjeux économiques du tourisme gourmand et ceux du positionnement des productions sur le marché de la consommation agroalimentaire, notamment du point de vue de leur exportation sur les marchés les plus lucratifs, sont toutefois de plus en plus difficiles à départager. Partout, producteurs et distributeurs cherchent à étayer l'argu- 
mentaire de la mise en marché, en tenant notamment compte de l'évolution des sensibilités des consommateurs. Or, en ce domaine, présence de traditions, beauté des lieux et caractère artisanal réel ou suggéré de la production constituent les trois piliers des stratégies.

Les dispositions prises par les vignerons pour embellir et protéger leurs domaines vont dans le même sens: le terroir, l'ensemble du contexte paysager dans lequel le vin s'élabore, est devenu argument de vente. (Pigeat, 2000: 16)

La reproduction, voire le constitution, d'un paysage typique permet non seulement d'assurer la qualité spécifique du produit, mais aussi la permanence de l'image associée à ce produit. (Cartier, 2004 : 4)

Le positionnement sur l'échiquier du tourisme gourmand et, par extension, sur celui du tourisme régional, agricole ou vert, rejoindrait, dans ses contradictions, le positionnement-marché de productions artisanales ou prétendues telles. L'un et l'autre joueraient du local pour mieux accéder au global. Or, n'est-il pas paradoxal d'observer que la recherche de distinction alimentée par la mise à plat des cultures gastronomiques locales en raison de la mondialisation passe par une offre touristique qui risque d'être banalisée à force d'être indistinctement reprise partout?

Il y a en effet dans le discours du marketing une opposition entre la quête du global et le fait de garder un cadre de référence local, opposition autour de laquelle se dessine [sic] non seulement l'ambiguité mais la fragilité de ce discours. (Procoli, 2003: 163)

\section{Conclusion}

La géographie du tourisme gourmand est une géographie de l'ambiguiité. Davantage évocatrice que fondatrice, elle s'articulerait pour une bonne part à une instrumentalisation du territoire d'emblée destinée à faire bonne impression chez ceux et celles qui souhaitent faire bonne chère. En mobilisant le concept de terroir, cette instrumentalisation de l'espace vise moins à inscrire les productions dans un cadre géohistorique précis qu'à conférer aux

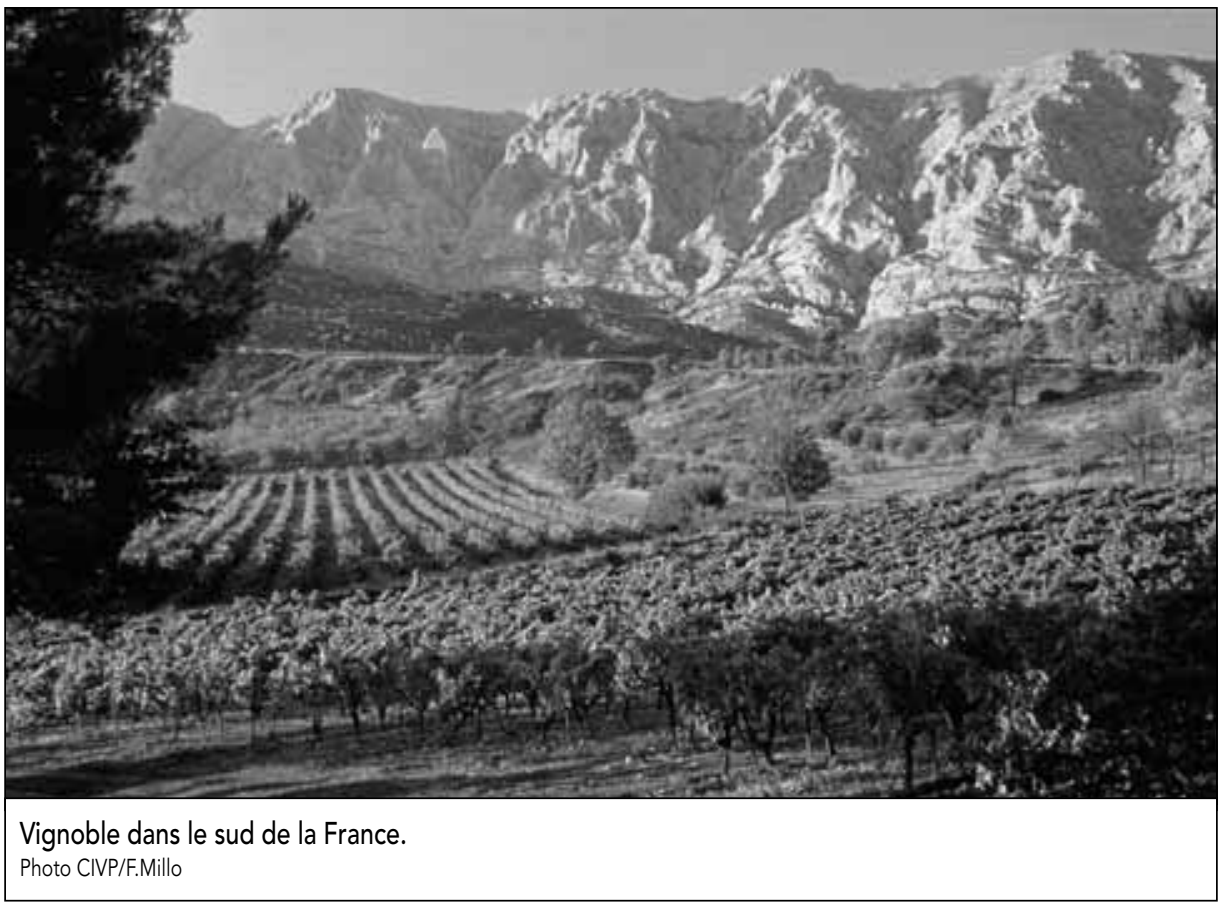

stratégies de marketing qui y prennent appui une certaine caution que peuvent renforcer les certifications destinées à protéger les appellations d'origine. Compte tenu des enjeux économiques en présence, on comprend que des promoteurs du tourisme gourmand puissent prendre quelque latitude à l'égard d'un concept qui les sert par ailleurs bien.

Gérard Beaudet est urbaniste, directeur de I'Institut d'urbanisme et chercheur associé à la Chaire en paysage et environnement de l'Université de Montréal.

\section{Bibliographie}

Caldicott, Chris et Carolyn, et Merrell James (2001) La route des épices, La Renaissance du livre, coll. "Art de vivre et saveurs gourmandes ", $192 \mathrm{p}$.

Cauquelin, Anne (1989) L'invention du paysage, Plon, $181 \mathrm{p}$.

Cartier, S. (2004) "Terroirs en nuances ", States, vol. 11, [http://strates.revues.org/document 396.html], $13 \mathrm{p}$.

Desjardins, A. (2005) «Toques et terroir », Le Soleil, 31 octobre.

Donadieu, P. (1998) Campagnes urbaines, Acte sud, $219 p$.

Donadieu, P. (1999) « Campagnes urbaines : de la réalité aux symboles ", dans P. PoullaouecGonidec, M. Gariépy et B. Lassus (dir.) Le paysage, territoire d'intentions, L'Harmattan, p. 79-93.
Dorion, J. (1999) Un dimanche à la campagne, Éditions du Trécarré, $159 \mathrm{p}$.

Dorion, J. (2003) Les terroirs du Québec : Guide agrotouristique, Éditions du Trécarré, $47 \mathrm{p}$.

Foglia, P. (2005) «T'as déjà vu une brebis, toi ? ", La Presse, 20 novembre, p. A2.

Luginbühl, Y. (1995) «Le paysage rural ; la couleur de l'agricole, la saveur de l'agricole, mais que reste-t-il de l'agricole? ", dans A. Roger (dir.), La théorie du paysage en France (1974-1994), Champ Vallon, p. 313-333.

Mayle, P. (1991) A Year in Provence, Vintage, $224 \mathrm{p}$.

Phaneuf, M. (2005) Voyageur du vin: Regard photographique sur les vignobles du monde, Les éditions de l'Homme, $143 \mathrm{p}$.

Pigeat, J.P. (2000), Les paysages de la vigne, Solar, $159 \mathrm{p}$.

Procoli, A. (2003) «La quête de 'pureté' et la fabrication de l'aliment de qualité. Le pâté de porc Hénaf", dans L.K. Morisset, P. Dieudonné et J.F. Simon (dir.) Réinventer pays et paysages, centre de recherche Bretonne et Celtique, p. 161-172. 